\title{
Analysis of China's Natural Gas Infrastructure Development Strategy
}

\subsection{Current Development of Natural Gas Infrastructure}

Since the West-East Pipeline began operating in 2004, China's natural gas infrastructure has developed rapidly. As of the end of 2014, a pipeline network distribution layout has been formed with the West-East Pipeline, marine gas and near-region supply, providing coverage to all provinces with the exception of Tibet. There are also both pipeline gas and LNG import resource channels, opening up central Asia's pipeline gas, central Myanmar's pipeline gas and marine LNG import channels. There is also underground gas storage and LNG receiving stations as two major peak shaving methods, covering the seaboard region, gas-producing regions and regions around the Bohai Sea.

\footnotetext{
* This chapter was overseen by Zhonghong Wang from the Development Research Center of the State Council and Shangyou Nie from Shell International Exploration and Production. It was jointly completed by Xiaowei Xuan, Yingxie Yang, Yongwei Zhang, Jiaofeng Guo, Weiming Li, Beiqing Yao, Tao Hong and Yuxi Li from the State Resource Department Oil and Gas Centre, Xiaoli Liu from the National Development and Reform Commission's Energy Office, Guang Yang and Jianhong Yang from the China Energy Research Institute's Natural Gas Centre, Xiaobo Ju and Beijing Yao from Shell China. Other members of the topic group participated in discussions and revisions.
}

\subsubsection{Current State of Infrastructure Development}

As of the end of 2014, China had constructed $82,000 \mathrm{~km}$ of natural gas pipeline, of which $23,300 \mathrm{~km}$ are national trunk pipelines, $16,500 \mathrm{~km}$ are national branch pipelines and 13,800 km are provincial trunk pipelines, with major progress achieved in terms of urban gas pipeline networks. In addition, $11 \mathrm{LNG}$ receiving stations have been built, with total receiving capacity of 39.40 million tons. Constructed underground gas storage bases number 19, with total warehousing capacity of 45.2 billion $\mathrm{m}^{3}$ and designed working gas volume of 15.1 billion $\mathrm{m}^{3}$, and effective working gas volume of 4.2 billion $\mathrm{m}^{3}$.

\section{Natural gas pipeline networks}

Since the 1960s when China built its first natural gas pipeline, the "Bayu Line", over many decades of development, China's natural gas pipelines have undergone significant growth. This is especially true with the 2004 West-East Pipeline, which accelerated China's natural gas pipeline construction. As of the end of 2014, China had the West-East Pipeline, ShaanxiBeijing Line, Sichuan to East Gas Pipeline Project, the Yuji Line and China-Myanmar natural gas pipeline as major national trunk pipelines, and the Jining Line, Zhongwu Line, Zhonggui Line and Huaiwu Line as major connection pipelines, with regional pipeline networks between the Yangtze River Delta, Sichuan and 
Chongqing, and North China. This has achieved a long-distance transport pipeline connectivity to major consumption markets, as well as to underground gas storage and LNG receiving stations, providing coverage to all provinces with the exception of Tibet and preliminarily forming a national natural gas grid.

\section{LNG receiving stations}

As of the end of 2014, China's operating, approved and under construction LNG receiving stations numbered 15 , of which 11 were currently in operation, and four had been approved and were under construction, located in over 10 provinces and regions including Guangdong, Fujian, Shanghai, Zhejiang, Huainan, Jiangsu, Liaoning, Shandong, Tianjin, and Tangshan. Currently operating LNG receiving stations have a receiving and unloading capability of 39.40 million tons per year, which is equivalent to 55 billion $\mathrm{m}^{3}$ of natural gas. In 2014, annual imported LNG resources amounted to 18.79 million tons, equivalent to 26.3 billion $\mathrm{m}^{3}$ of natural gas (Figs. 9.1 and 9.2).

\section{Underground storage}

As of 2014, China had constructed the following underground storage facilities: Lamadian North, Dazhangtuo, Ban 876, Banzhong North, Ban 808, Ban 828, Jinyun, Jing 58 Storage, Wen 96, Suqiao, Xiangguo Temple, Hutubi, Shuang 6 and Bannan. These were primarily distributed in eight provinces-Jiangsu, Tianjin, Hebei, Liaoning, Heilongjiang, Xinjiang, Chongqing and Henan - with total designed capacity of 45.2 billion $\mathrm{m}^{3}$ and designed total working gas volume of 15.1 billion $\mathrm{m}^{3}$ and effective working gas volume of 4.2 billion $\mathrm{m}^{3}$ (Fig. 9.3).

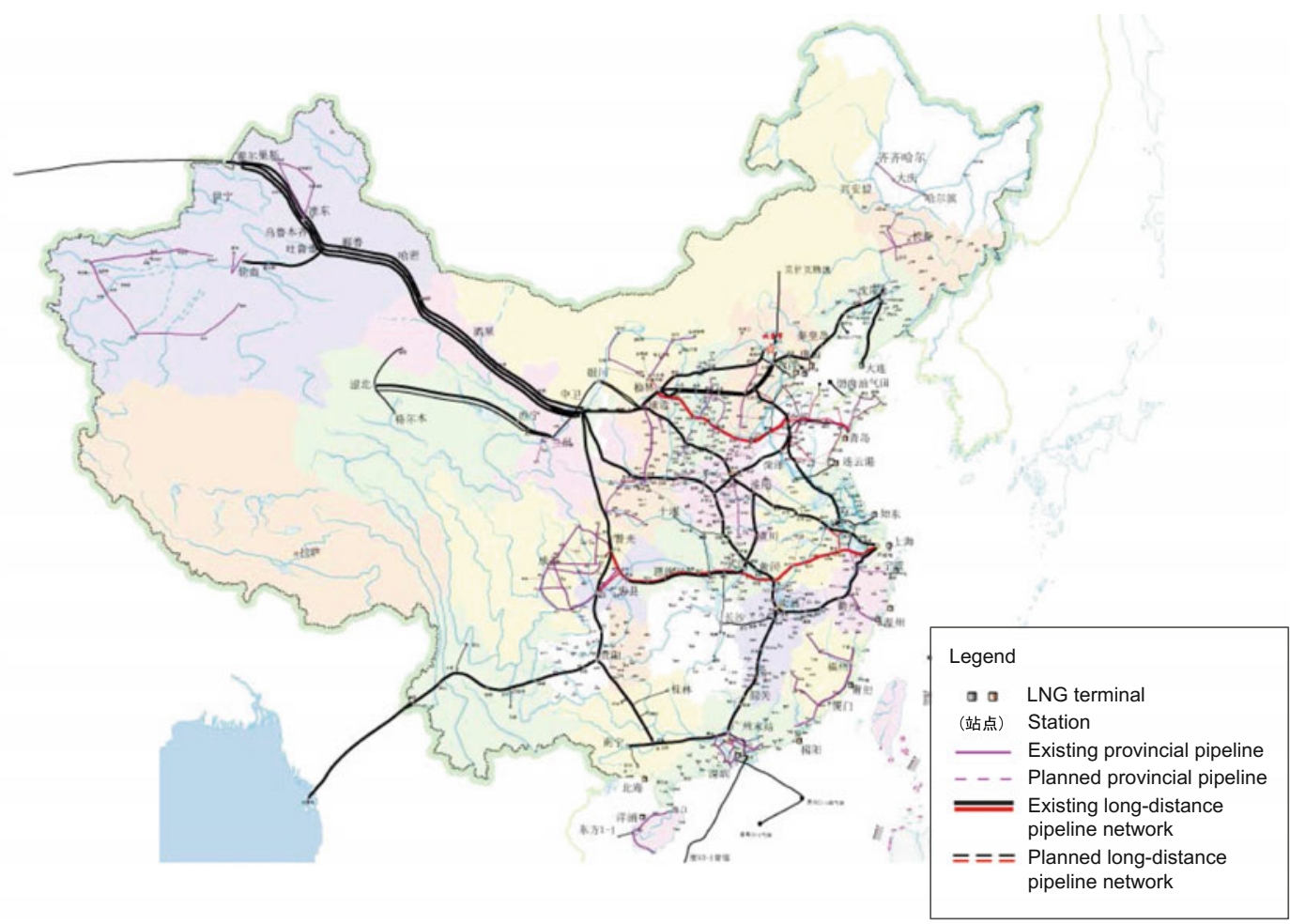

Fig. 9.1 China's natural gas pipeline network distribution 


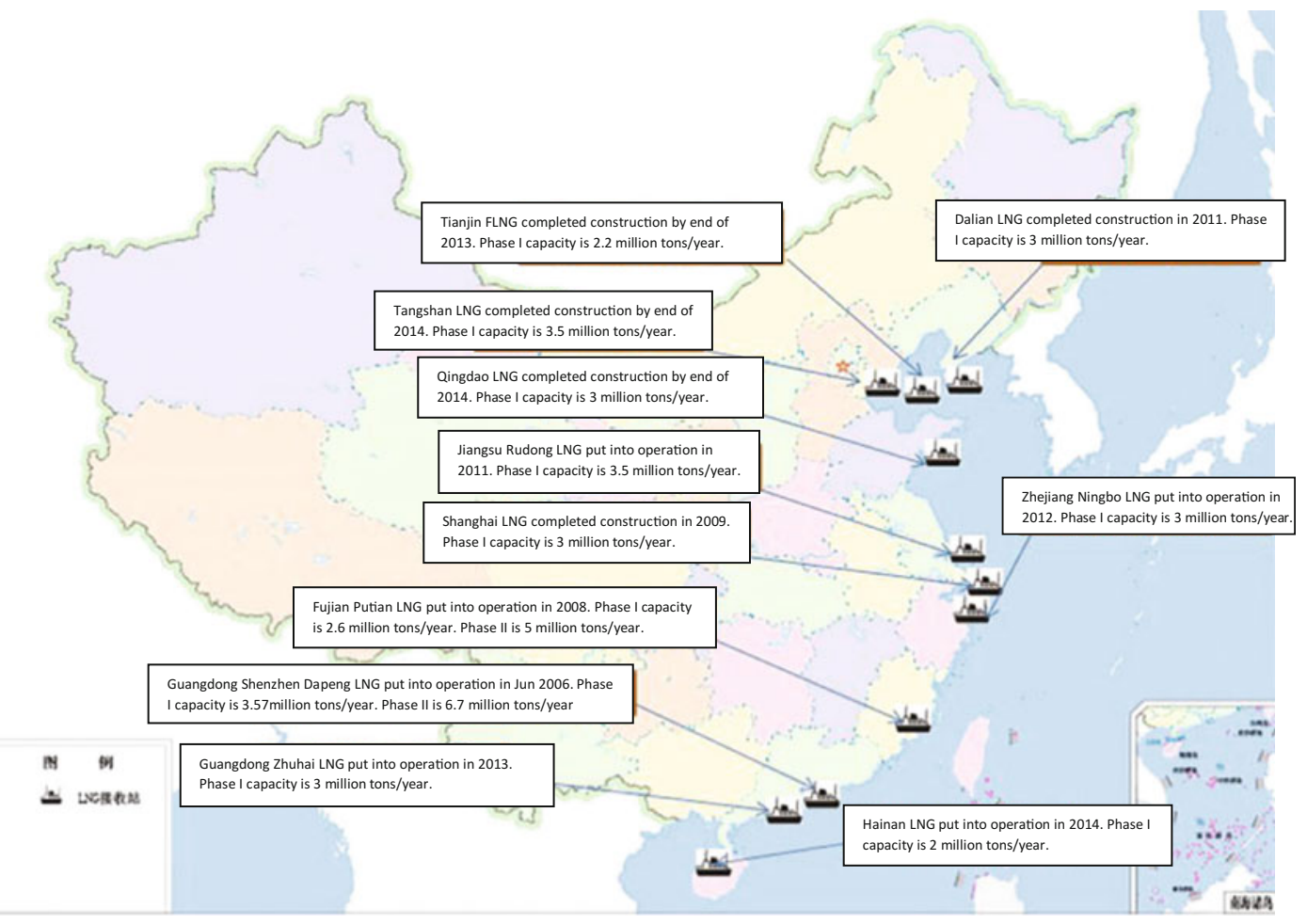

Fig. 9.2 LNG receiving station distribution map

\subsubsection{Assessment of Development Levels and Existing Problems}

\section{Pipeline construction capacity shortcomings}

With the completion and operation of the West-East pipeline in 2004, China's natural gas pipeline length reached $25,000 \mathrm{~km}$. In 2010 , after the West-East Pipeline 2 and the Sichuan to East Pipeline, China's natural gas pipeline length reached $56,000 \mathrm{~km}$. As of the end of 2014 , pipeline network length was $82,000 \mathrm{~km}$, with natural gas consumption volume having reached 183 billion $\mathrm{m}^{3}$. According to the natural gas industry development experience of the United States, when consumption volume is 130 billion $\mathrm{m}^{3}$, pipeline length was $175,000 \mathrm{~km}$.
Whether it is in terms of satisfying China's natural gas usage demands or when compared to the history of developing nations, China's natural gas pipeline construction is markedly underdeveloped.

As of the end of 2014, China's natural gas pipeline network distribution capabilities were 240 billion $\mathrm{m}^{3}$, with natural gas consumption exceeding 128 billion $\mathrm{m}^{3}$. Based on market consumption volume of 1.2 fold as a peak shaving demand, the required pipeline distribution capacity was 220 billion $\mathrm{m}^{3}$. From this it is clear that the current pipeline network distribution capabilities can only meet the market demand of the next several years, and if future natural gas market development is to be supported, accelerated promotion of pipeline network facility construction will be required (Fig. 9.4). 


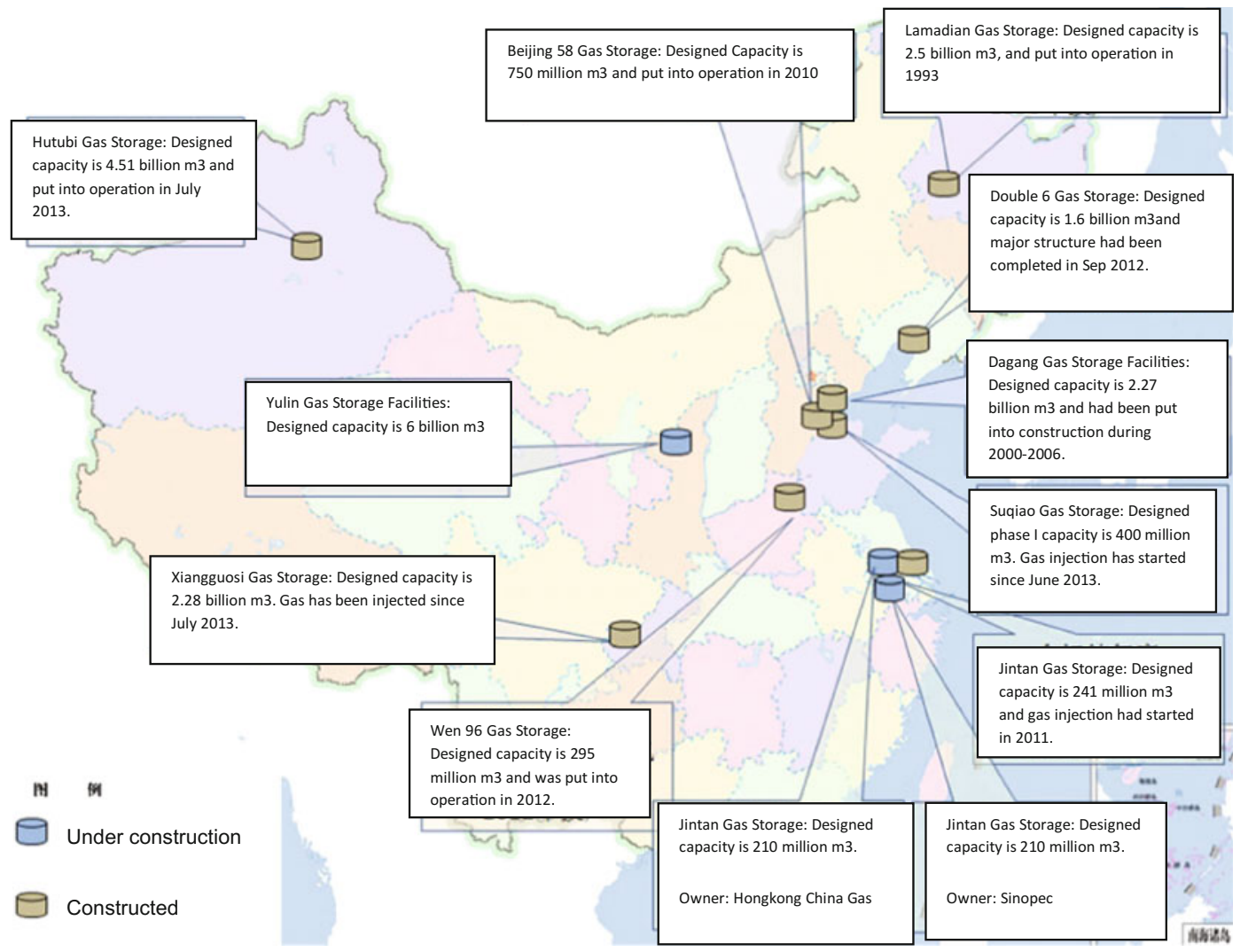

Fig. 9.3 Underground gas storage distribution

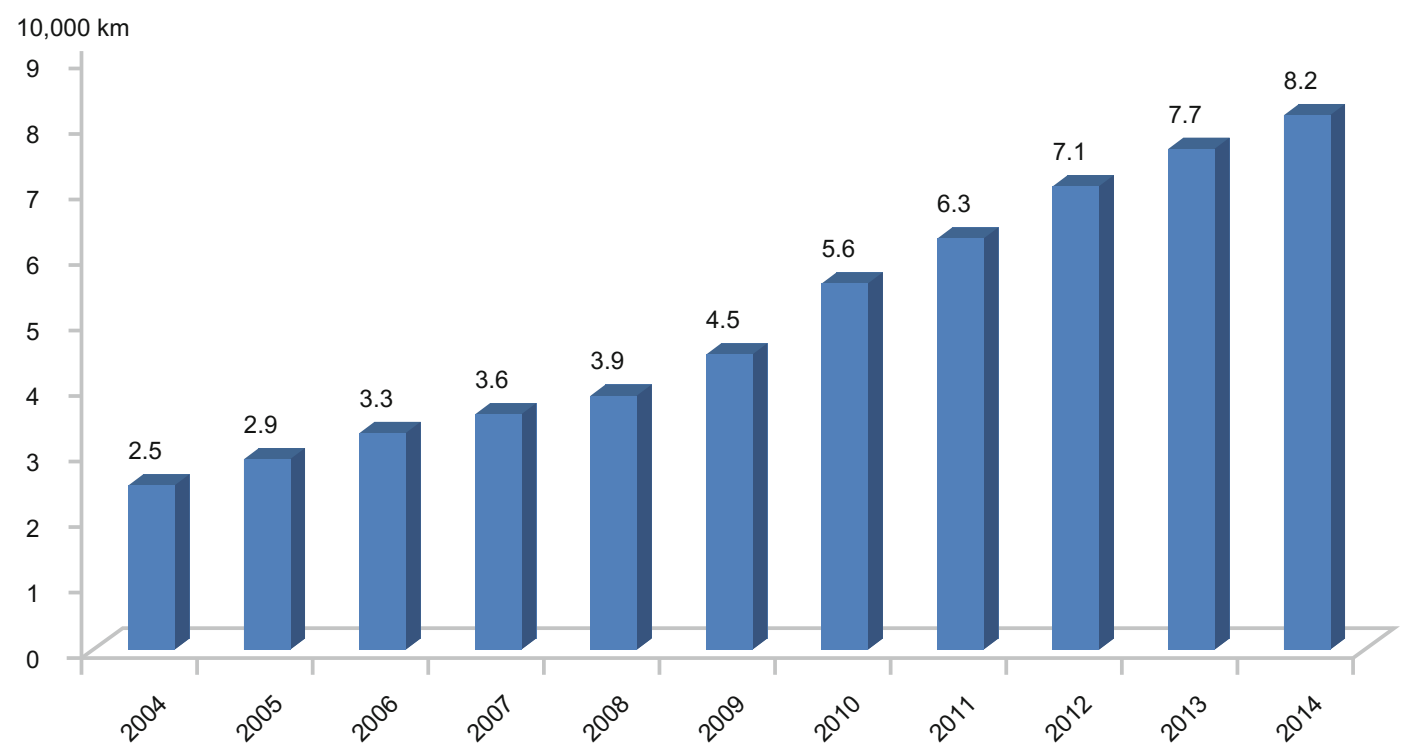

Fig. 9.4 China's natural gas pipeline construction 


\section{Development of LNG infrastructure}

Since 2006 when the Dapeng LNG facility was completed, China's LNG receiving station construction speed has gradually accelerated. As of the end of 2014, constructed and operating LNG receiving stations numbered 11 in total, with receiving and offloading capacity of 39.40 million tons per year, equivalent to 55 billion $\mathrm{m}^{3}$ of natural gas.

Because current LNG receiving stations primarily satisfy city demand in a radius around the receiving station itself, and each region's natural gas development has had a relatively long and staged development, LNG receiving stations are quick to enter production once they are completed. Also, because of regional preferential development of urban fuel, and limited increases in volume, in order to resolve the lack of market space for LNG receiving stations as a result of their rapid growth, accompanying fuel gas plants have been established to consume the exceed gas volume. As markets continue to develop, urban gas demands are gradually growing, and price tolerance is higher than power plants, making it easy for a power plant to have ample gas volume in early stages of development of a receiving station, but once the market develops to need LNG as a major gas source, the power plants could see supply shortages.

Therefore, China's current LNG receiving stations have played an important role in increasing supply capacity, and their strong abilities in terms of peak shaving offer critical effects in maintaining secure and stable operation of the market. However, their rapid growth can lead to uneven development of natural gas infrastructure.

\section{Shortage of underground gas storage peak shaving capability}

China's natural gas peak shaving method is primarily based on underground gas storage. Location is very particular for gas storage, with long construction cycles and a significant quantity of gas required to build the reserve. Underground gas storage has high requirements for geological conditions, and it is difficult to find suitable locations. Salt cavern underground storage facility construction periods are long, for example the Jinyun underground storage location took over 10 years from commencement of construction to operation. In addition, underground gas storage requires significant input for the initial reserve, and with a designed gas volume of 200 million $\mathrm{m}^{3}$, approximately 100 million $\mathrm{m}^{3}$ are required as an initial reserve.

China's research and construction of underground storage began relatively late, and there is a relatively major gap as compared to developed nations. According to operational experience in foreign markets, if natural gas market operational risk remains at relatively low levels, then the storage dissipation ratio (referring to the ratio between natural gas peak shaving volume and natural gas demand volume) should reach $15 \%$. In 2014, China's natural gas apparent consumption reaches 183 billion $\mathrm{m}^{3}$, with underground storage peak shaving volume of 4.2 billion $\mathrm{m}^{3}$, making peak shaving volume account for less than $3 \%$ of total natural gas consumption, thus meaning that peak shaving capabilities are severely lacking.

\subsection{Opportunities and Challenges}

\subsubsection{The Energy Development Strategy Action Plan (2014-2020)}

The Energy Development Strategy Action Plan proposed major development of natural gas, with non-renewable energy consumption totals to be controlled by 2020 to within 4.8 billion tons of standard coal, and natural gas to account for over $10 \%$ of non-renewable energy consumption. Based on principles of developing land and marine resources and conventional and unconventional sources, acceleration of conventional natural gas production would be sought, resolving development battlements as quickly as possible in unconventional natural gas, thus prompting natural gas reserve production volume growth. Based on the gas supply layout involving the West-East Pipeline, the North to South Gas Pipeline and the Sea to Land Pipeline, natural gas 
pipeline and reserve facilities were to be constructed more rapidly, thereby forming a national natural gas trunk pipeline network connecting production regions and consumptions regions. There was a clear proposal to reach over $120,000 \mathrm{~km}$ of natural gas trunk pipeline by 2020.

\subsubsection{Policy Catalyses Rapid Development of the Natural Gas Industry}

"Several Opinions Regarding Establishing an Effective Mechanism to Ensure Stable Natural Gas Supply" proposes acceleration of the development of natural gas supply, with 400 billion $\mathrm{m}^{3}$ of natural gas supply capability by 2020 and striving to reach 420 billion $\mathrm{m}^{3}$, supporting the progress of the "Coal to Gas" project. Further support is proposed for various market entities to participate equally in gas reserve facility investment, construction, and operation, with various regions to strengthen their gas reserve peak shaving facilities, LNG receiving stations, and storage facilities. "Guiding Opinions of the NDRC Regarding Acceleration of Progress in Gas Reserve Construction" proposes strengthening the sense of urgency regarding gas reserve facility construction, speeding up in-progress construction and encouraging various ownership models for construction and operation of gas reserves. Gas reserve facilities are the guarantee for safe and stable natural gas operation, and the nation supports various entities in participating in gas reserve facility construction, operation and management, promoting the construction of such facilities.

\subsubsection{The Atmospheric Pollution Prevention Action Plan}

In order to deal with atmospheric pollution and to improve air quality, the Atmospheric Pollution Prevention Action Plan was formulated, which proposes an acceleration of clean energy substitution, raising supplies of natural gas, coal natural gas and coalbed methane. By 2015, newly added natural gas trunk line pipeline capabilities will reach over 150 billion $\mathrm{m}^{3}$, offering coverage to Beijing-Tianjin-Hebei, the Yangtze River Delta and the Pearl River Delta. Optimisation of natural gas usage methods will be overseen, adding new natural gas that preferentially ensures residential usage or substitution of coal, thus encouraging development of natural gas distributed energy and other highly efficient utilisation projects while limiting the development of natural gas chemical engineering projects. This approach enables an orderly development of natural gas peak shaving power stations. Infrastructure for natural gas is an important assurance for market development, and the powerful implementation of "Coal to Gas" has accelerated efforts in infrastructure construction.

\subsubsection{Existing Pipeline Network Capabilities Are Insufficient}

According to resource support and market demand analysis, it is expected that by 2020 , China's natural gas market demand for natural gas will be 400 billion $\mathrm{m}^{3}$, and by 2030 it will be 600 billion $\mathrm{m}^{3}$. If 1.1 times the demand volume can provide resource supply assurance, demand volume of 1.15 times shipping capabilities can assure market distribution. By 2020, pipeline network distribution capabilities should reach 260 billion $\mathrm{m}^{3} /$ year, and 690 billion $\mathrm{m}^{3} /$ year in 2030 . As of the end of 2014, China's resource distribution pipeline network capabilities were 240 billion $\mathrm{m}^{3}$, only satisfying $52 \%$ of distribution demand expected for 2020 and $35 \%$ that of 2030 . There is a need to accelerate promotion of infrastructure construction to satisfy the needs of market demand.

\subsubsection{Peak Shaving Capabilities Are Severely Lacking}

Calculating based on $15 \%$ at the storage dissipation ratio, in 2020 China's natural gas market demand will be 400 billion $\mathrm{m}^{3}$, and peak shaving demand will be 60 billion $\mathrm{m}^{3}$. By 2030, peak shaving demand will be 90 billion $\mathrm{m}^{3}$. Currently, 
underground storage peak shaving capabilities are only at 4.2 billion $\mathrm{m}^{3}$, and LNG receiving stations have peak shaving capability of 2.6 billion $\mathrm{m}^{3}$, calculated based on 0.2 times that steady distribution of gas at market peak shaving. From this it is clear that current peak shaving facility capabilities are far from adequate to meet future natural gas market peak shaving demand.

\subsubsection{Increasing Pressure for Safe Operations}

As natural gas markets continue to grow, infrastructure construction is likewise building, and there is increasing pressure for safe and steady operations. Currently, China's trunk pipelines continue to use a point-to-point resource supply, with different operators lacking interconnectivity between their pipelines. Provincial trunk line investment entities are more diversified, and pipeline supplies do not intersect. At peak gas periods, resource allocation is impossible, and when a pipeline network encounters malfunction there is no effective energy response approach. In future, China's natural gas trunk pipelines should establish interconnectivity and provincial trunk lines should be freely allocable.

\subsection{Natural Gas Infrastructure Development Strategy}

China's overall natural gas business and policy guidance has formulated natural gas development objectives, proposing a natural gas infrastructure development strategy to guide the rapid development of natural gas infrastructure.

\subsubsection{Guiding Considerations}

Taking the safe and stable operation of national pipeline networks as a target function, infrastructure construction should be planned to achieve diversified supply. The aim should be to construct flexible allocation infrastructure systems, strengthening gas pipeline hub construction, speeding up peak shaving facility construction, establishing interconnectivity and securing gas supply systems that can be flexibly allocated. Modern natural gas industry systems need to be constructed, with stable supply, efficient operation and co-ordinated upstream, midstream and downstream development, and a national unified infrastructure development strategy needs to be established.

\subsubsection{Development Objectives}

Bearing in mind the guiding considerations for natural gas infrastructure development, and in the context of China's natural gas industry development, the natural gas infrastructure development goals for China by 2030 are as follows:

- Achieve interconnected gas supply systems for various operators at top-tier natural gas pipelines. Pipeline network length will reach $150,000 \mathrm{~km}$ by 2020 , and gas delivery capability will exceed 460 billion $\mathrm{m}^{3}$. 2030 will see $250,000 \mathrm{~km}$ of pipeline network and gas delivery capability of over 690 billion $\mathrm{m}^{3}$.

- Achieve a four-pronged layout that includes West-East Pipeline, North to South Pipeline, Sea to Land Pipeline, and local supply. Optimise the natural gas supply and allocation stations in Ningxia Zhongwei, Hubei, Hebei Yongqing, Shanghai and Guangdong Wuqu. Construct six major underground reserve groups in the central plains region, northern China, the north-east, in Changqing, in the north-west and in the south-west. Aim to achieve 62 billion $\mathrm{m}^{3}$ of peak shaving capability by 2020 , with an effective underground reserve working volume of 44 billion $\mathrm{m}^{3}$. Achieve 90 billion $\mathrm{m}^{3}$ of peak shaving capability by 2030, and an effective underground reserve working volume of 77 billion $\mathrm{m}^{3}$.

- Establish four major LNG import channels based on north-west China-Asia imports, south-west China-Myanmar imports, northern China-Russia imports and eastern imports. From 2020 to 2030, achieve 85 billion $\mathrm{m}^{3}$ of distribution capability on the China-Asia 
Lines A, B, C and D, with 12 billion $\mathrm{m}^{3}$ of capability on the China-Myanmar line, 3868 billion $\mathrm{m}^{3}$ on the China-Russia line, and 100 million tons of receiving capability through LNG imports.

\subsubsection{Development Strategy}

In order to guarantee that China's natural gas infrastructure is designed for safe and stable operation, interconnected systems between the nation's various entities involved in natural gas infrastructure should be firmly established. To strengthen infrastructure construction capabilities, there should be overall planning for facility construction, building up diversity of investment and implementation. Finally, in order to improve infrastructure usage efficiency, fair third-party access operation and management platforms should be created.

As natural gas market demand further grows, China's natural gas infrastructure must be further optimised, and by 2020 important pipeline construction must be completed, including the ChinaRussia natural gas pipeline, the West-East Pipeline 3, West-East Pipeline 4, West-East Pipeline 5, Shaanxi-Beijing, Xinjiang coal methane pipeline, Erdos coal methane, coastal arteries and the Sichuan-Chongqing-Hubei natural gas pipeline. There is also a need to bring national pipelines to $150,000 \mathrm{~km}$, with long-distance distribution pipelines to reach 44,000 km. By 2030, based on market demand, efforts will intensify in large pipeline network deployments, with the newly added West-East Pipeline 5, West-East Pipeline 6, the China-Russia east-west pipeline and construction of at least $250,000 \mathrm{~km}$ of national pipelines with long-distance distribution of up to $60,000 \mathrm{~km}$ and gas distribution capabilities to exceed 690 billion $\mathrm{m}^{3}$.

The ultimate goal in achieving interconnectedness between different operating entity infrastructure is to achieve secure and stable operations, reasonable resource allocation and connection between resources and markets and between gas reserves and markets. The implications of interconnectivity between the infrastructure of various operating entities are: (1) national long-distance trunk pipelines are interconnected through hub stations and connection lines; (2) provincial trunk pipelines are freely allocated through split hubs; (3) underground gas reserves and LNG receiving stations are connected to national long-distance trunk pipelines; and (4) neighbouring provincial and municipal markets form a regional network.

\section{National long-distance trunk pipeline interconnectivity}

China's long-distance trunk pipelines are primarily constructed by CNPC and Sinopec, with CNOOC primarily constructing marine gas shore connection and LNG receiving station external distribution pipelines. Long-distance trunk pipeline interconnectivity is not only to achieve connection with internal pipelines of oil companies, but also to achieve connection with pipelines between oil companies. National long-distance trunk pipeline interconnectivity relies on pipelines connected by central hubs and through connection lines.

Forming central hubs: Based on China's current natural gas infrastructure development and future infrastructure development, central hubs are to form in Ningxia Zhongwei, Hebei Yongqing, Hubei, Shanghai and Guangdong. As part of this:

- Ningxia Zhongwei is a point of coalescence between western resources and eastern distribution, sending western gas to eastern distribution systems, and connecting to the Xinjiang coal methane pipelines and Shaanxi Beijing line systems, the Zhongwei-Jingbian pipeline, the Zhonggui Line and other pipelines. This achieves connection between imported China-Asia gas, Xinjiang gas fields, Changqing gas fields and Xinjiang coal methane.

- Hebei Yongqing is the hub for northern China, and connects the Shaanxi-Beijing line, the China-Russia natural gas pipeline, the north China gas reserve groups and the Tianjin-Hebei resource pipeline. In northern China it adjusts natural gas supply, optimising natural gas resource allocation and relieving demand for gas at peak periods.

- Hubei has many national long-distance trunk pipeline channels, and is the point of 
coalescence for the central and southern region hubs, forming a connection with Xinjiang conventional gas, Xinjiang coal methane, imports from the China-Asia line, imports from the China-Russia line, Sichuan-Chongqing conventional gas and Sichuan-Chongqing shale natural gas.

- Shanghai is China's natural gas market centre, and from the perspective of supply structure, Shanghai is the central hub for West-East Line 1 and 2, the Sichuan to East pipeline and other important natural gas lines while also receiving East Sea gas and importing a large volume of LNG. From its characteristics, Shanghai is a natural gas trading centre but also a national natural gas pricing baseline location, and in future will become an important natural gas hub for China.

- As an important resource hub, Guangdong is set to become a point of integration for multiple gas sources. From the perspective of supply structure, there is pipeline gas, marine gas and LNG, with pipeline supply including the West-East Line2, the Xin'aozhe line, the West-EastLine3, marine gas and multiple LNG receiving stations. From the perspective of properties, supply resources include conventional natural gas, coal methane and LNG. With a variety of resources coming together, it is a complex province with national gas sources.

Construction of connection lines: Connection lines are important complementary resource bridges between national long-distance trunk pipelines, with constructed examples being the Cheng Zhong Gui Line, the Huai Wu Line and the Ji Ning Line. Of these, the Zhong Gui connects the West-East Pipeline, Changqing gas fields, Sichuan-Chongqing gas region and the China-Myanmar pipeline resources. The Huai Wu line connects the West 1 Line, West 2 Line and the Zhong $\mathrm{Wu}$ Line. The Ji Ning Line connects the Shaanxi-Beijing Line, the West 1 Line and the Rudong LNG resources.

China's coastal LNG receiving stations provide supply essentially point-to-point, with supply regions and peak shaving capabilities being limited. Moving forward, the focuses of construction for connection lines will be along coastal arteries, with pipelines to connect receiving stations from Liaoning Dalian LNG to Guangxi's North Sea LNG receiving stations, with intermediary LNG receiving stations, marine gas to shore pipelines and the transited national trunk pipelines (Fig. 9.5).

\section{Provincial trunk line free allocation}

China's various provincial natural gas infrastructure entities are diverse, with national enterprises and private corporations competing with one another, and without interconnected business communications, which causes overlapping construction of infrastructure, low usage efficiency and an inability to optimally allocate resources. Provincial natural gas infrastructure must form a unified management and operations platform, carrying out unified planning and construction for provincial infrastructure while unifying operations and management, resource co-ordination and distribution. Provincial trunk line interconnectivity is required to achieve flexible allocation of resources and prefecturelevel dual gas source supply deployment.

Current provinces in China that have only one provincial natural gas company include: Beijing, Tianjin, Hebei, Jiangsu, Shanghai, Zhejiang, Guangdong, Shaanxi, Anhui, Inner Mongolia, Hubei and Chongqing. Provinces with two or more provincial natural gas companies include: Shanxi, Jiangxi and Hunan. Each provincial natural gas company's business model can be categorised as: unified purchase of resources, agent distribution, mixed operation, monopolistic construction of infrastructure and free construction. In order to realise the goals of provincial pipeline unification planning, provincial trunk line free allocation and provincial market balanced development, each province should actively establish provincial natural gas companies, with unified operation and management from the provincial natural gas companies, whose business models should be divided into two categories:

- The first category includes provincial trunk line unified planning, staged implementation and unified purchase of resources. In order to avoid overlapping infrastructure construction 


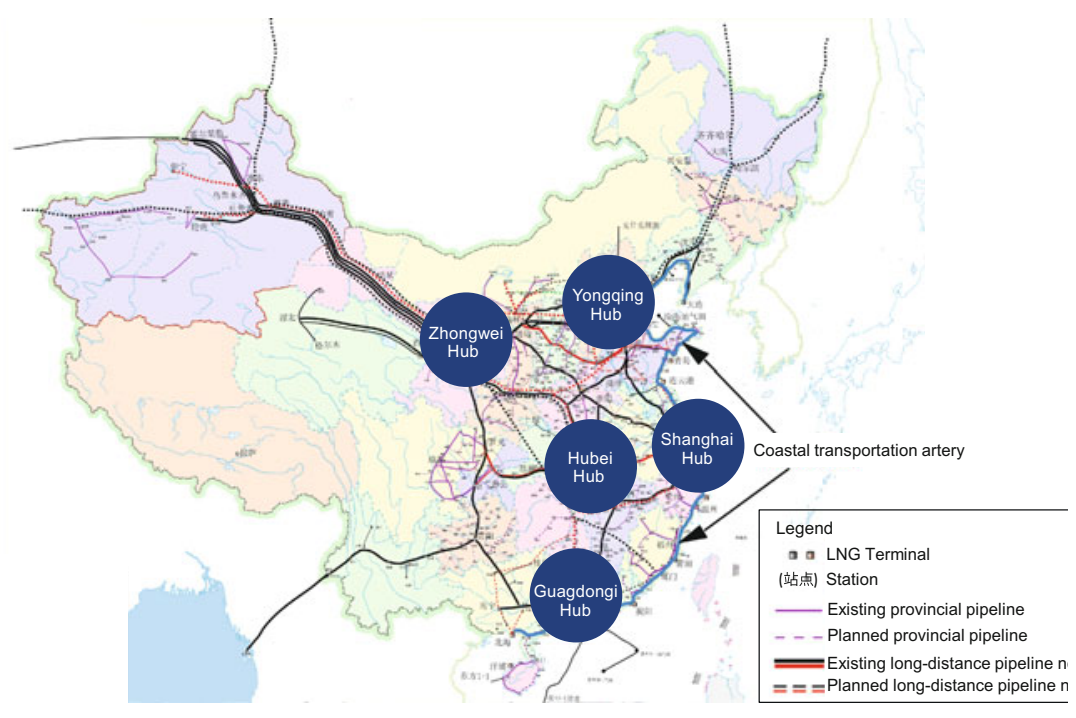

Fig. 9.5 Interconnectivity between various entity natural gas infrastructures across the country

and the resulting inefficiencies, there should be overall planning for provincial natural gas infrastructure. Based on the degree of market development, staged implementation should take place, with provincial trunk lines and peak shaving and allocation facilities constructed and operated by provincial natural gas companies. Resource procurement should be procured in unison by the provincial natural gas companies, with the natural gas resources that provincial users require negotiated with the provincial natural gas company and sold at a standard price that is not differentiated by distance.

- When infrastructure construction stages are in line with the above, but provincial companies do not carry out resource procurement and sales, the downstream users carry out negotiations directly with upstream gas suppliers, and gas suppliers then distribute the natural gas through the provincial natural gas companies. The provincial natural gas companies collect a management and distribution fee, and downstream users pay resource fees to the upstream gas supplier and a distribution fee to the provincial natural gas company. To safeguard the interests of users relatively distant from long-distance pipelines, provincial natural gas companies provide a uniform price to users within the province.

\section{Underground gas reserves and LNG receiv- ing station peak shaving co-ordination}

Currently, China has two major large peak shaving facilities. One is underground gas reserves, and the other is LNG receiving stations. Of these, the underground gas reserves are distributed across the mainland and are distributed via pipeline. For these, the peak shaving principle is to adjust between long-distant and nearby pipelines. LNG receiving stations are distributed along the coastline, with their peak shaving principle being adjustment of nearby supplies and liquid adjustments. After 2020, underground reserve peak shaving will be the primary approach, with auxiliary peak shaving from LNG receiving stations.

Two types of peak shaving facilities: Currently, China's natural gas peak shaving facility capabilities are limited and are far from being able to satisfy future development demands, given that peak shaving capabilities should reach 62 billion $\mathrm{m}^{3}$ by 2020 and effective underground gas reserve volume will need to be 44 billion $\mathrm{m}^{3}$. By 2030, peak shaving capabilities must reach 90 billion $\mathrm{m}^{3}$, with effective underground reserve volume of 77 billion $\mathrm{m}^{3}$.

Based on China's current natural gas circumstances, in future the country's newly built underground gas reserves will primarily rely upon exhausted oil and gas deposits. By 2030 a 
group of six reserves will be formed in the central plains region, northern China, the north-east, in Changqing, in the north-west and in the south-west. As part of this, the central plains reserve group connects the Yu Ji Line, Xin'aozhe $\mathrm{Yu} \mathrm{Lu} \mathrm{Sub-Trunk} \mathrm{Line} \mathrm{and} \mathrm{the} \mathrm{West} 2$ Line Tai'an Sub-Trunk Line. The northern China group connects the Shaanxi-Beijing Line and the China-Russia import pipelines. The north-east group connects the China-Russia import pipelines. The Changqing group connects the Shaanxi-Beijing Line and the West-East Pipeline. The north-west group connects to the West-East Pipeline. The south-west group connects the Zhong Gui Line and shale natural gas external distribution pipelines.

LNG receiving stations are connected through coastal arteries, and exchange resources with coastal region natural gas markets during peak shaving. Using tanker trucks for external distribution, peak shaving is provided for urban fuel and enterprise LNG reserve gas.

Regional peak shaving method distribution and options: Reserve facilities for natural gas are an important measure used to ensure the safe and stable supply of natural gas, and form an integral component part of natural gas delivery systems. Currently, the construction of gas reserve capability is lagging seriously behind, and according to national natural gas pipeline network layouts, the construction of such facilities should be accelerated, aiming to ensure that natural gas peak shaving emergency response requirements are met. In long-distance pipelines, details must be determined according to specific local needs, ensuring reasonable deployments, clear focal points and staged implementation of accessory construction of peak shaving facilities.

There was relatively early construction of gas reserve facilities in provinces and cities, including Beijing, Tianjin, Hebei, Shanxi, Liaoning, Jilin, Heilongjiang and Shandong, and these regions have good foundations. Moving forward, the focus will be on gradual optimisation of existing gas reserves and newly built underground reserves, complemented by small and medium LNG facilities and LNG receiving station storage. In conjunction with existing gas storage facilities, there will be optimised construction within exhausted oil deposits in Liaohe, Dagang, North China, Daqing and Shengli, including Liaohe Shuang 6, Qi 13, Shengliyong 21, Dagangban South, North China Su 1, Gong 20, Su 4, Su 49, Guxinzhuang, Wen 23, Saqing and Jilin oil fields.

Gas reserve facility conditions are relatively poor in Shanghai, Jiangsu and Zhejiang. Priority should be given to constructing LNG reservoirs, with underground storage and small and medium reserves as auxiliary peak shaving systems. Primary projects include the Jiangsu salt cavern gas reserve and the Jiangsu oil field exhausted oil deposit gas reserve. Prior to 2015, the focus had been on LNG gas reserves, relying on Jiangsu and Zhejiang existing LNG receiving station expansions to grow LNG reserves, thereby forming an LNG gas reserve system comprising Jiangsu and Zhejiang.

Fujian, Guangdong, Guangxi, Hainan and Yunnan have focused on LNG receiving station reserves, with small and medium storage and underground gas reserves, as well as small and medium liquefaction facilities providing auxiliary support. This region is working to construct an LNG reserve system prior to 2015 that is based on the LNG receiving station expansions in Fujian, Guangdong and Hainan, so as to satisfy regional peak shaving demand. Prior to 2030, once a reasonable network has been established, new LNG receiving stations will be constructed to increase gas reserve capabilities, while at the same time building a certain amount of underground gas reserve working gas volume, thereby establishing multiple interrelated peak shaving measures that satisfy local gas reserve system needs and the needs of the surrounding region.

Anhui, Hubei and Hunan have a certain amount of advantageous geography, and can mainly construct underground gas reserves, with auxiliary peak shaving systems of LNG small and medium reserves and small and medium liquefaction facilities. Major projects include Hubei Yingcheng, Yunying and Huangchang salt cavern gas reserves.

Shanxi, Henan, and Sichuan must use exhausted oil and gas deposits to build 
underground gas reserves while at the same time using upstream gas fields to resolve a portion of peak shaving problems. This is supplemented by peak shaving of users that can have supplies halted and small and medium liquefaction facility peak shaving. Major projects include Zhongyuanwen 23, Zhongyuanwen 96 and South-West Xiangguosi, among other exhausted oil and gas deposit gas reserves.

Shaanxi, Gansu, Qinghai, Ningxia, and Xinjiang are focused primarily on underground gas reserves for their gas reserve systems, building gas reserves from exhausted oil and gas deposits, including in Xinjiang Hutubi and Yulin.

\section{Regional networks between provinces}

Due to administrative zoning and approval procedures, regional pipeline networks are primarily concentrated within provinces (with the exception of long-distance pipelines and connection lines that run between provinces), resulting in relatively large differences in natural gas consumption between neighbouring provincial economies. In future, government guidance and support for construction of trunk line connections between provinces will allow resource supplementation, and thus boost the development of regional natural gas markets and optimise the regional pipeline network systems.

\subsection{Standardising Infrastructure Planning and Diversifying Investment}

\subsubsection{Infrastructure Construction Planning and Project Progress Oversight}

Infrastructure development involves foreign import pipelines, LNG receiving stations, national trunk long-distance pipelines, provincial pipelines and underground gas reserves, among other facilities. As part of this, pipeline network construction is critical to developing the natural gas market, but currently China's natural gas infrastructure is primarily constructed and operated by three large oil companies that have not established effective interconnectivity. As infrastructure continues to be optimised and interconnection of infrastructure deployments becomes critical to ensuring the secure and stable operation of the national natural gas network, effective infrastructure interconnection will become increasingly important, with the construction of Xin'aozhe, the West 4 Line, West 5 Line, China-Russia East-West Line, coastal receiving stations and underground gas reserves. Only with effective co-ordination in planning stages to form a complete industry chain can effective infrastructure connectivity be realised. Therefore, government intervention is required to create entities to oversee infrastructure construction and to strengthen oversight and controls of such development.

The major reason for a low degree of infrastructure planning is the lack of review mechanisms for planning construction projects. There is planning for projects, but no subsequent follow-up regarding progress in project implementation. A review needs to be carried out of enterprises involved in the planning of infrastructure projects at the national level, reviewing project implementation progress and timeline goal completion, taking measures to reward and penalise as required. A review should also be carried out for departments involved in provincial infrastructure, with consistent review criteria; provincial departments should actively implement relevant work involving the planning of provincial projects, working hard to co-operate and creating review systems for units under their supervision to promote active construction of infrastructure and optimise the outcomes of the practical work.

\subsubsection{Promote Construction of Entities and Investment Diversification}

The country encourages and supports various forms of capital participation and investment structure in the central planning of natural gas 
infrastructure. The three major oil companies should release investment and construction rights to national trunk pipelines, introducing private capital. Some of the provincial natural gas company monopolies on provincial infrastructure construction need to be broken. The introduction of private capital to infrastructure construction can increase participation in construction and improve the capacity for it.

The construction of CNPC West 3 Line brought together equity from the national social insurance fund council, an urban infrastructure industry investment fund and Baosteel Group, diverging from the previous model of investment contribution that was monopolised by CNPC's own investments and for the first time allowing private capital to be included in natural gas long-distance pipeline construction. The planned West 4 Line, West 5 Line and Xin'aozhe, among other national natural gas trunk pipelines, should carry out joint investment construction with private capital, based on the West 3 Line model. The uniformity seen in national long-distance pipeline construction entities has not yet been disrupted completely, though, and entities outside the three major oil companies should be introduced to diversify investment in the nation's long-distance pipelines.

Provinces with provincial natural gas companies should co-operate actively in investments with qualified private capital, accelerating the construction of provincial infrastructure. Province sub-trunk pipeline construction rights should be fully opened, and permission should be granted for qualified enterprises to construct and operate infrastructure within the ambit of national and local government planning.

\subsection{Establishing Fair Third-Party Access to Infrastructure}

\subsubsection{Create the Conditions for Third-Party Access}

As at the end of 2014, China had put into operation 16 natural gas national trunk pipelines, over 80 national sub-trunk pipelines, 11 LNG receiving stations and 19 underground gas reserves. There are no statistics available regarding the operating conditions of most infrastructure. By using open and fair operating systems, it would become possible to learn about infrastructure operating conditions, and excess capabilities could be put to use. When natural gas pipeline network facility operators have excess pipeline network facility capabilities, they can fairly open their pipeline network facilities to third parties, providing delivery, storage, gasification, liquefaction and compression services. In this mutually beneficial scenario, good use of can be made of facility capabilities while ensuring that existing users continue to have access to services, with a preferential order based on signed contracts with newly added users so as to realise fair, non-discriminatory open usage of natural gas pipeline network facilities.

Considering the fact that third-party access conditions are not yet mature, barriers between companies are difficult to eliminate, though some suggestions to catalyse third-party access are as follows:

\section{- Construct third-party review institutions} to fairly and equitably verify excess infrastructure capabilities: Considering the lack of transparency regarding infrastructure excess capabilities and the difficulties involved in downstream user verification, which result from asymmetry in information, third-party independent verification institutions should be established in order to fairly and equitably verify oil and gas pipeline infrastructure, storage, gasification, liquefaction and compression excess capability.

- Pipeline network independence: Currently, infrastructure enterprises provide transmission, storage, gasification, liquefaction and compression services while at the same time having businesses such as exploration and sales. This has resulted in local or partial monopolies within the natural gas industry at its various stages of development. Partition production shipping and sales business. At 
the sales stage, break the monopolies to achieve highly efficient infrastructure usage. At the same time, due to the uniqueness of natural gas shipping and the heavy reliance on pipeline networks, separate national pipeline networks into several independent pipeline networks, with competition and co-operation between and among them to achieve highly efficient infrastructure utilisation.

- Enterprise establishment and implementation measures: On June 24, 2014, CNPC reviewed and passed in principle the CNPC Natural Gas Group Oil and Gas Pipeline Network Infrastructure Open Access Implementation Measures (Pilot), stipulating that oil and gas pipeline network facilities be fairly opened, as well as assigning management responsibilities to the relevant departments. Each enterprise should establish and implement measures based on its own infrastructure operating capabilities, operating costs and operating scope, including time used, scope and price.

- Infrastructure interconnectivity, fee rates and liberalised market operation: Through connectivity between pipelines and other infrastructure, break through natural gas transmission stage monopolies. Pipeline transmission fees should be liberalised in their operation, with the market to determine final resource allocation.

\subsubsection{Establish Open and Transparent Oil and Gas Management and Operation Release Platforms}

According to the Natural Gas Development Twelfth Five-Year Plan, natural gas infrastructure interconnectivity and services for third-party access should be implemented. At the end of the Twelfth Five-Year Plan, China will achieve partial connectivity of its pipelines, and have the conditions in place to achieve third-party access.

The utilisation of excess infrastructure capability cannot be entirely resolved simply by establishing operating entities, and each facility's operations can only be fully understood by the operating entity, with third parties unable to obtain information about them. Therefore, open and transparent oil and gas management and operation release platforms must be established, to cover all existing excess capacity in the infrastructure, with operators allowing third-party access to terms and fees, and operator-related contact information. Third parties should carry out specific negotiations with operators based on their needs. Because the status of infrastructure operators can be variable, they should provide the latest data relating to their infrastructure operations to the platforms, and the platform can then provide timely updates that are publicly available.
Open Access This chapter is licensed under the terms of the Creative Commons Attribution 4.0 International License (http://creativecommons.org/licenses/by/4.0/), which permits use, sharing, adaptation, distribution and reproduction in any medium or format, as long as you give appropriate credit to the original author(s) and the source, provide a link to the Creative Commons license and indicate if changes were made.
The images or other third party material in this chapter are included in the chapter's Creative Commons license, unless indicated otherwise in a credit line to the material. If material is not included in the chapter's Creative Commons license and your intended use is not permitted by statutory regulation or exceeds the permitted use, you will need to obtain permission directly from the copyright holder. 